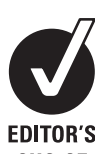

CHOICE

${ }^{1}$ Department of Ophthalmology, Medicine of Sensory Function, Ehime University Graduate

School of Medicine, Toon-City,

Ehime, Japan; ${ }^{2}$ Division of

Ophthalmology, Minami-

Matsuyama Hospital,

Matsuyama-City, Ehime, Japan

Correspondence to:

Dr S Kawasaki, Department of Ophthalmology, Ehime University

School of Medicine, Shitsukawa,

Toon-City, Ehime 791-0295,

Japan; skawasak@m.ehime-u. ac.jp

Accepted 13 April 2009

Published Online First

10 June 2009

\title{
Evaluation of filtering bleb function by thermography
}

\author{
S Kawasaki, ${ }^{1}$ S Mizoue, ${ }^{1}$ M Yamaguchi, ${ }^{1}$ A Shiraishi, ${ }^{1}$ X Zheng, ${ }^{1}$ Y Hayashi, ${ }^{2}$ Y Ohashi ${ }^{1}$
}

\section{ABSTRACT}

Aim: To determine whether thermography can be used to evaluate bleb function.

Methods: The surface temperatures of filtering blebs from 39 eyes of 33 post-trabeculectomy patients were measured using thermography. We introduced a new parameter: the temperature decrease in the filtering bleb (TDB) where TDB $=$ (mean temperature of the temporal and nasal bulbar conjunctiva)-(temperature of the filtering bleb). The eyes were classified into good and poor intraocular pressure (IOP) control groups according to the patients' postoperative IOP. The morphological appearance of the filtering blebs were classified by slit-lamp images according to the Indiana Bleb Appearance Grading Scale. The differences in TDB were analysed statistically. Results: Mean overall scores were as follows. The TDB were $0.54(S D 0.20)^{\circ} \mathrm{C}$ and $0.21(S D 0.18)^{\circ} \mathrm{C}$ for the good and poor IOP control groups, respectively. The difference between the TDB of the two IOP control groups was significant $(p<0.0001)$, but there was no significant relationship between morphological classification and TDB.

Conclusions: Thermographic measurements suggest that functional blebs have lower temperatures than nonfunctional blebs, and their morphological appearances are not affected by their temperature. Thermography may be useful for the evaluation of bleb function.

Trabeculectomy is widely performed as a reliable surgical procedure for reducing intraocular pressure (IOP). In postoperative management, slit-lamp biomicroscopy is routinely used to check bleb function. Generally, filtering blebs are classified into three categories: cystic, diffuse and flat blebs. ${ }^{12}$ A more precise classification system has been introduced recently. ${ }^{3}$ The clinical features of well or poorly functioning blebs are well described. ${ }^{4-6}$ However, evaluating bleb function by slit-lamp is not always accurate nor easy. To overcome this problem, the internal structure of filtering blebs has been examined by ultrasound biomicroscopy (UBM), ${ }^{7}$ optical coherence tomography (OCT $)^{9-11}$ and in vivo confocal microscopy (Rostock cornea module). ${ }^{12}{ }^{13}$ In spite of such advanced morphological methods, there are still limitations in accuracy and reliability.

Essentially, in a well functioning bleb, aqueous humour flows out of the scleral flap to perfuse the subconjunctival space. We hypothesised that differences in aqueous humour dynamics in filtering blebs might be reflected as differences in surface temperature. Therefore, we tried to measure the temperature of filtering blebs by thermography. Thermography is a non-invasive technique used to measure the surface temperature of an object by detecting the intensity of infrared light that is emitted from the object. It was introduced into the medical field in 1957 by Barnes ${ }^{14}$ and has been used in ophthalmology since the mid 1960s, ${ }^{15}$ particularly for the diagnosis of dry eye syndrome and other ocular surface disorders. ${ }^{16-23}$ However, the temperature of a filtering bleb has never been measured before. In this study, we examined the relationship between bleb function and the temperature of the filtering bleb.

\section{MATERIALS AND METHODS \\ Study protocol}

Thirty-five consecutive patients (41 eyes of 20 men and 15 women) were studied between September 2005 and September 2006. Two patients were excluded from the study because full exposure of the filtering bleb was difficult due to extremely narrow lid fissures and conjunctival scarring, respectively. Therefore, 39 eyes were analysed over 6 months after the last trabeculectomy.

Surgeries were performed in the Ophthalmology Division of Minami Matsuyama Hospital between November 2000 and February 2006. In all cases, a surgical sponge soaked with $0.04 \%$ mitomycin C was applied around the site of the scleral flap for 3 min, followed by rinsing with $200 \mathrm{ml}$ of balanced salt solution (Alcon, Fort Worth, Texas, USA).

The type of glaucoma, age, preoperative IOP, postoperative IOP, duration from surgery to thermography, location of the conjunctival flap, and morphological appearance of the filtering bleb were recorded for each patient.

\section{Measurement of ocular surface temperature by thermography}

A non-contact thermographic device (TH1106; NEC San-ei Instruments, Tokyo, Japan) was used. This instrument can detect temperatures ranging from $-10^{\circ} \mathrm{C}$ to $70^{\circ} \mathrm{C}$, with a minimum temperature resolution of $0.025^{\circ} \mathrm{C}$. One second was required to perform each measurement. The temperature data were transformed into a colourcoded image that was displayed in real time on a monitor with a resolution of $256 \times 207$ pixels. Its focal range was set from $20 \mathrm{~cm}$ to infinity. Using the highest magnification, the ocular surface was measured in one frame, $30.0 \mathrm{~mm} \times 28.4 \mathrm{~mm}$, so that $1 \mathrm{~mm}^{2}$ was equal to $8 \times 8$ pixels when the instrument was positioned $20 \mathrm{~cm}$ from the ocular surface. The thermographic device was calibrated using a black body radiator on a regular maintenance schedule.

Each subject was examined in a room at 26.0 (SD $1.5)^{\circ} \mathrm{C}$ and 40.0 (SD 5.0)\% humidity, with standard indoor levels of illumination and no air drafts. We measured the ocular surface temperature after the subject had rested for $15 \mathrm{~min}$ in a natural state, according to Kabayama's technique. ${ }^{16}$ The thermographic device was set up $20 \mathrm{~cm}$ in front of the eye, 
and the head was held steady by a head-holder frame. First, the subject was instructed to open his eyes naturally and look straight ahead, and after a blink, a measurement was made. Then, the examiner raised the upper eyelid and instructed the subject to look downward to expose the bleb, and another measurement was made within $1 \mathrm{~s}$. The temperature was measured within $1 \mathrm{~s}$ of opening the eye, because ocular surface temperature is known to change during eye opening. ${ }^{21}$ Thus the measurement taken at the time point immediately after eyelid opening was thought to reflect most accurately the surface temperature of bleb when it was covered by the eyelid. All thermographic measurements were performed in a special examination room used only for thermography by one examiner who was not given any information about the subjects' IOP control.
To establish the location of the bleb in reference to the cornea and conjunctiva, slit-lamp digital photographs were taken at the same time and the same magnification as the thermograms. Each region of interest, corresponding to the centre of the cornea, the temporal and nasal bulbar conjunctiva, and the filtering bleb, was electronically outlined with a box measuring $16 \times 16$ pixels. The computer determined the mean value for the pixels. The temperatures of the temporal and nasal bulbar conjunctiva were determined at the midpoint between the corneal limbus and either the medial or lateral canthus, and the temperature of the filtering bleb was determined at the centre of the scleral flap.

Comparing temperatures as absolute values may not be appropriate, because body temperature varies among individuals. To resolve this problem, we introduced a new parameter:

Table 1 Detailed patient information

\begin{tabular}{|c|c|c|c|c|c|c|c|c|c|c|c|c|c|}
\hline \multirow[b]{2}{*}{ Case } & \multirow{2}{*}{$\begin{array}{l}\text { IOP } \\
\text { control* }\end{array}$} & \multirow{2}{*}{$\begin{array}{l}\text { Type of } \\
\text { glaucoma }\end{array}$} & \multirow[b]{2}{*}{ Sex } & \multirow{2}{*}{$\begin{array}{l}\text { Age } \\
\text { (years) }\end{array}$} & \multirow{2}{*}{$\begin{array}{l}\text { Preop IOP } \\
\text { (mmHg) }\end{array}$} & \multirow{2}{*}{$\begin{array}{l}\text { Postop IOP } \\
\text { (mmHg) }\end{array}$} & \multirow{2}{*}{$\begin{array}{l}\text { Duration from } \\
\text { surgery to } \\
\text { thermography } \\
\text { (months) }\end{array}$} & \multirow{2}{*}{$\begin{array}{l}\text { Base of the } \\
\text { conjunctival flap }\end{array}$} & \multicolumn{4}{|c|}{ IBAGS grade } & \multirow[b]{2}{*}{ TDB $\left({ }^{\circ} \mathrm{C}\right.$} \\
\hline & & & & & & & & & H & $\mathbf{E}$ & V & $\mathbf{S}$ & \\
\hline 1 & Good & POAG & $\mathrm{F}$ & 83 & 37 & 14 & 12 & Limbal & 2 & 1 & 1 & 0 & 0.55 \\
\hline 2 & Good & POAG & $\mathrm{F}$ & 83 & 30 & 15 & 6 & Limbal & 2 & 2 & 1 & 1 & 0.30 \\
\hline 3 & Good & POAG & $\mathrm{F}$ & 79 & 17 & 11 & 12 & Fornix & 1 & 1 & 1 & 0 & 0.55 \\
\hline 4 & Good & POAG & $\mathrm{F}$ & 77 & 24 & 13 & 16 & Fornix & 1 & 2 & 3 & 0 & 0.35 \\
\hline 5 & Good & POAG & $\mathrm{F}$ & 74 & 17 & 13 & 49 & Limbal & 2 & 3 & 1 & 0 & 0.45 \\
\hline 6 & Good & POAG & $\mathrm{F}$ & 74 & 35 & 12 & 14 & Fornix & 2 & 2 & 2 & 0 & 0.45 \\
\hline 7 & Good & POAG & $\mathrm{F}$ & 74 & 24 & 9 & 14 & Fornix & 2 & 2 & 2 & 0 & 0.25 \\
\hline 8 & Good & POAG & $\mathrm{F}$ & 67 & 33 & 11 & 12 & Fornix & 2 & 2 & 0 & 1 & 0.70 \\
\hline 9 & Good & POAG & $M$ & 83 & 20 & 13 & 20 & Fornix & 2 & 2 & 0 & 0 & 0.60 \\
\hline 10 & Good & POAG & $M$ & 62 & 31 & 13 & 26 & Limbal & 3 & 3 & 1 & 0 & 0.45 \\
\hline 11 & Good & POAG & $\mathrm{M}$ & 55 & 16 & 13 & 7 & Limbal & 2 & 2 & 1 & 0 & 0.70 \\
\hline 12 & Good & POAG & $\mathrm{M}$ & 55 & 16 & 14 & 6 & Limbal & 2 & 1 & 1 & 1 & 0.40 \\
\hline 13 & Good & PEG & $\mathrm{F}$ & 74 & 18 & 13 & 6 & Limbal & 0 & 0 & 2 & 0 & 0.85 \\
\hline 14 & Good & PEG & $\mathrm{F}$ & 74 & 18 & 13 & 6 & Limbal & 0 & 0 & 3 & 0 & 0.95 \\
\hline 15 & Good & PEG & $\mathrm{F}$ & 70 & 24 & 10 & 13 & Limbal & 2 & 2 & 3 & 0 & 0.20 \\
\hline 16 & Good & PEG & $\mathrm{M}$ & 85 & 20 & 7 & 37 & Limbal & 1 & 1 & 2 & 0 & 0.45 \\
\hline 17 & Good & PEG & $\mathrm{M}$ & 84 & 25 & 12 & 6 & Limbal & 0 & 0 & 3 & 0 & 0.40 \\
\hline 18 & Good & PEG & $\mathrm{M}$ & 70 & 35 & 8 & 54 & Limbal & 1 & 3 & 0 & 0 & 0.45 \\
\hline 19 & Good & PEG & $\mathrm{M}$ & 67 & 28 & 13 & 6 & Limbal & 0 & 0 & 3 & 0 & 0.95 \\
\hline 20 & Good & SG-n & $\mathrm{F}$ & 72 & 38 & 8 & 43 & Fornix & 2 & 2 & 1 & 0 & 0.70 \\
\hline 21 & Good & SG-n & $\mathrm{F}$ & 66 & 47 & 10 & 14 & Fornix & 3 & 2 & 1 & 0 & 0.55 \\
\hline 22 & Good & SG-u & $\mathrm{F}$ & 65 & 24 & 16 & 29 & Limbal & 3 & 2 & 1 & 0 & 0.70 \\
\hline 23 & Good & SG-n & $M$ & 70 & 28 & 16 & 6 & Limbal & 3 & 3 & 0 & 1 & 0.50 \\
\hline 24 & Poor & POAG & $M$ & 82 & 19 & $16 \dagger$ & 28 & Fornix & 1 & 2 & 3 & 0 & 0.25 \\
\hline 25 & Poor & POAG & $M$ & 76 & 28 & $14 \dagger$ & 12 & Limbal & 1 & 2 & 0 & 1 & 0.30 \\
\hline 26 & Poor & POAG & $M$ & 76 & 18 & $17 \dagger$ & 40 & Limbal & 1 & 1 & 0 & 0 & 0.55 \\
\hline 27 & Poor & POAG & $M$ & 74 & 18 & $16 t$ & 43 & Limbal & 1 & 1 & 0 & 0 & 0.45 \\
\hline 28 & Poor & POAG & $M$ & 69 & 28 & $16 \dagger$ & 24 & LImbal & 0 & 0 & 3 & 0 & 0.20 \\
\hline 29 & Poor & POAG & $M$ & 48 & 35 & $14 \dagger$ & 55 & Limbal & 1 & 2 & 2 & 0 & 0.05 \\
\hline 30 & Poor & PEG & $\mathrm{M}$ & 81 & 30 & $15 \%$ & 33 & Fornix & 0 & 0 & 2 & 0 & -0.05 \\
\hline 31 & Poor & PACG & $\mathrm{F}$ & 69 & 22 & $17 t$ & 27 & Fornix & 0 & 0 & 2 & 0 & 0.05 \\
\hline 32 & Poor & POAG & $\mathrm{F}$ & 77 & 22 & $21 \div$ & 60 & Limbal & 1 & 0 & 3 & 0 & 0.15 \\
\hline 33 & Poor & POAG & $M$ & 72 & 25 & $23 t$ & 12 & Limbal & 1 & 1 & 2 & 0 & 0.10 \\
\hline 34 & Poor & POAG & $M$ & 70 & 42 & $23 t$ & 11 & Fornix & 1 & 1 & 3 & 0 & 0.00 \\
\hline 35 & Poor & POAG & $\mathrm{M}$ & 66 & 19 & $24 t$ & 43 & Limbal & 2 & 3 & 0 & 0 & 0.15 \\
\hline 36 & Poor & PEG & $\mathrm{M}$ & 79 & 28 & $23 \dagger$ & 6 & Limbal & 3 & 2 & 3 & 0 & 0.40 \\
\hline 37 & Poor & PEG & $\mathrm{M}$ & 75 & 26 & $21 \dagger$ & 12 & Fornix & 1 & 1 & 1 & 1 & 0.05 \\
\hline 38 & Poor & SG-i & $\mathrm{F}$ & 48 & 46 & $24 t$ & 19 & Limbal & 3 & 3 & 1 & 0 & 0.20 \\
\hline 39 & Poor & SG-n & $\mathrm{F}$ & 40 & 42 & $26 \div$ & 6 & Limbal & 3 & 3 & 1 & 0 & 0.50 \\
\hline
\end{tabular}

E, extent; H, height; IBAGS, Indiana Bleb Appearance Grading Scale; PACG, primary angle-closure glaucoma; PEG, pseudoexfoliation glaucoma; POAG, primary open-angle glaucoma; S, Seidel test; SG, secondary glaucoma caused by neovascularisation of the angle (-n), uveitis (-u), or the iridocorneal endothelial syndrome (-i); TDB, temperature decrease in the filtering bleb: $\mathrm{V}$, vascularity.

${ }^{*}$ Good: IOP $<21 \mathrm{mmHg}$ without antiglaucoma medications; poor: filtering blebs with antiglaucoma medications for elevated IOP.

†With two topical medications; $\$$ with three topical medications. 

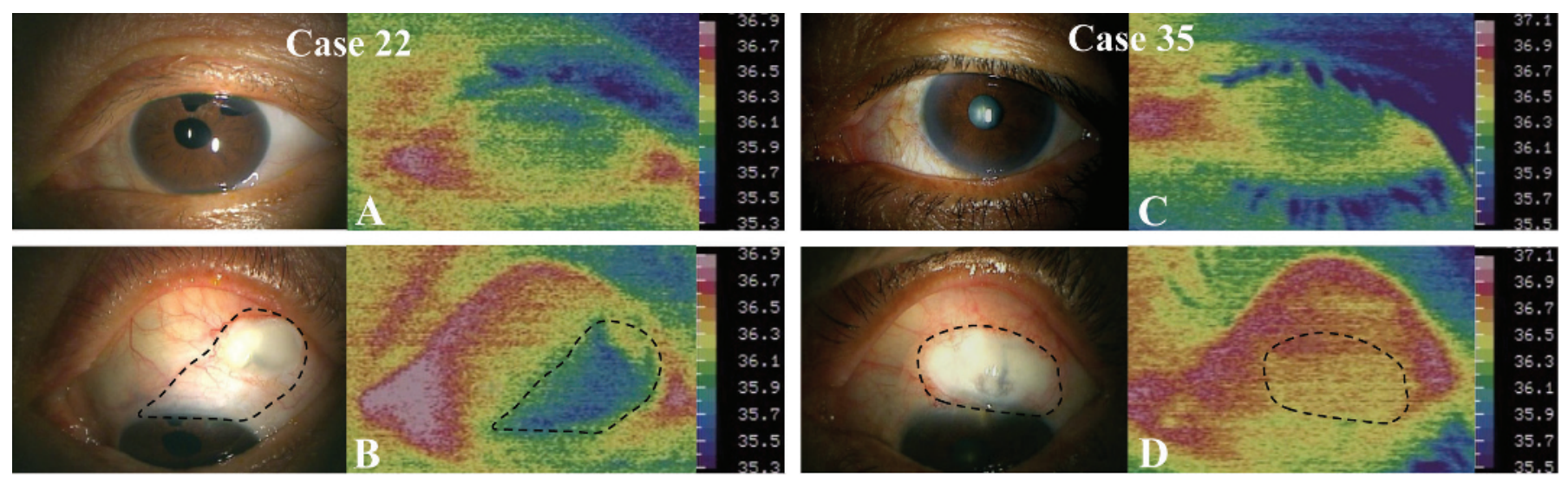

Figure 1 Representative slit-lamp photographs and thermograms from the good and poor intraocular pressure control group are shown. Dotted lines are drawn along the edge of the filtering bleb. Case $22(\mathrm{~A}, \mathrm{~B})$ belongs to the good IOP control group and case 35 (C, D) belongs to the poor IOP control group. Each thermogram is divided into eight different colours in $0.2^{\circ} \mathrm{C}$ steps from dark blue to pink. In the forward gaze $(A, C)$, the cornea is displayed as blue or green and the temporal and nasal bulbar conjunctiva as red or pink. In the downward gaze, the filtering bleb of the good IOP control group (case 22; $(B)$ ) is shown as green or blue, meaning that its temperature is clearly lower than that of the surrounding bulbar conjunctiva. Meanwhile, the filtering bleb of the poor IOP control group (case 35; (D)) is represented as yellow or pink, meaning that its temperature is almost same as that of the surrounding bulbar conjunctiva.

the temperature decrease in the filtering bleb (TDB). The temperature of the upper conjunctiva was assumed to be the original temperature of the filtering bleb. Therefore, the mean temperature of the temporal and nasal bulbar conjunctiva was substituted for the temperature of the upper conjunctiva. TDB is described by the equation $\mathrm{TDB}=$ (mean temperature of the temporal and nasal bulbar conjunctiva)-(temperature of the filtering bleb).

\section{Classification of subjects according to IOP control}

Filtering blebs were classified by IOP control according to criteria from previous studies. ${ }^{12}$ Functioning blebs, the good IOP control group, were defined as those with an IOP $<21 \mathrm{mmHg}$ without antiglaucoma medications, and failed blebs, the poor IOP control group, were defined as those who had received more than two eye drops of antiglaucoma medications for elevated IOP $\geqslant 21 \mathrm{mmHg}$ in the past. The differences between the two groups were examined.

\section{Morphological assessments}

It is clinically important to examine whether TDB is affected by the morphological appearance of the filtering bleb. Filtering blebs were classified by slit-lamp examination according to the Indiana Bleb Appearance Grading Scale (IBAGS); ${ }^{3}$; blebs were graded for height ( $\mathrm{H} 0-3)$, extent (E0-3), vascularity (V0-4), and leakage with the Seidel test (S0-2) in a masked manner. We examined the relationship between the morphological appearance and temperature of the filtering blebs.

Table 2 Mean temperatures and statistical analyses of the good and poor intraocular pressure control groups

\begin{tabular}{llllll}
\hline $\begin{array}{l}\text { IOP } \\
\text { control } \\
\text { group }\end{array}$ & Cornea & $\begin{array}{l}\text { Temporal } \\
\text { conjunctiva }\end{array}$ & $\begin{array}{l}\text { Nasal } \\
\text { conjunctiva }\end{array}$ & $\begin{array}{l}\text { Filtering } \\
\text { bleb }\end{array}$ & TDB \\
\hline Good & $35.45(0.53)$ & $35.87(0.48)$ & $36.01(0.48)$ & $35.40(0.50)$ & $0.54(0.20)$ \\
Poor & $35.53(0.50)$ & $35.92(0.43)$ & $36.05(0.42)$ & $35.78(0.43)$ & $0.21(0.18)$ \\
p Value & 0.6063 & 0.6991 & 0.8523 & $0.0307^{*}$ & $<0.0001^{*}$ \\
\hline
\end{tabular}

IOP, intraocular pressure; TDB, temperature decrease in the filtering bleb. Values are mean (SD).

*Mann-Whitney U test.

\section{Statistical analyses}

The $\chi^{2}$ test, Fisher's exact test, Mann-Whitney $U$ test or ANOVA were used to determine the statistical significance of any differences. The level of significance was set at $p<0.05$. Pearson's correlation coefficient was used for examining correlation, and intraclass correlation coefficients were used for examining the reproducibility of our thermographic measurements.

\section{RESULTS}

The details of the subjects are summarised in table 1 . The mean age of the subjects at the time of thermography was 70.9 (SD 10.4) (range 40-85) years. There were 23 filtering blebs in the good IOP control group and 16 filtering blebs in the poor IOP control group. No significant differences between the two groups were found in the type of glaucoma ( $p=0.5193, \chi^{2}$ test), age $(p=0.6675$, Mann-Whitney $U$ test), preoperative IOP ( $p=0.4656$, Mann-Whitney $U$ test), time from surgery to thermography ( $p=0.1270$, Mann-Whitney $U$ test) or site of the base of the conjunctival flap ( $p>0.9999$, Fisher's exact test).

\section{Reproducibility}

The reproducibility of our thermographic measurements was examined. Thermography was performed three times at intervals of more than $1 \mathrm{~min}$ in 11 eyes in forward gaze and 22 eyes in downward gaze that were selected randomly from the subjects. The intraclass correlation coefficients for the temperature at the centre of the cornea, the temporal bulbar conjunctiva, the nasal bulbar conjunctiva and the filtering bleb were $0.9748,0.9811,0.9768$ and 0.9680 , respectively.

\section{Correlation between IOP reduction and TDB}

In this study, there were 13 well-functioning blebs that received full antiglaucoma medications (three or more eyedrops with oral acetazolamide therapy) before trabeculectomy (cases 1, 2, 4, $6-8,10,15,17,18,20,21$ and 23). We examined the correlation between IOP reduction and TDB in these 13 cases. The mean IOP reduction was 20.9 (SD 7.4) $\mathrm{mmHg}$ and the mean TDB was $0.43(\mathrm{SD} 0.15)^{\circ} \mathrm{C}$. The two factors were correlated (Pearson's correlation coefficient: $0.6822, p=0.010196)$. 
Figure 2 Representative thermograms of both cystic and flat blebs in the good and poor intraocular pressure control groups are shown. Dotted lines are drawn along the edge of the filtering bleb. Cases 10 and 19 belong to the good IOP control group and cases 34 and 39 belong to the poor IOP control group. In the good IOP control group, the temperature of the filtering bleb is clearly lower than that of the surrounding bulbar conjunctiva, even if no cystic bleb is recognised in the slitlamp examination. In the poor IOP control group, the temperature of the filtering bleb is almost the same as that of the surrounding bulbar conjunctiva, even if a filtering bleb with a cystic appearance is present.
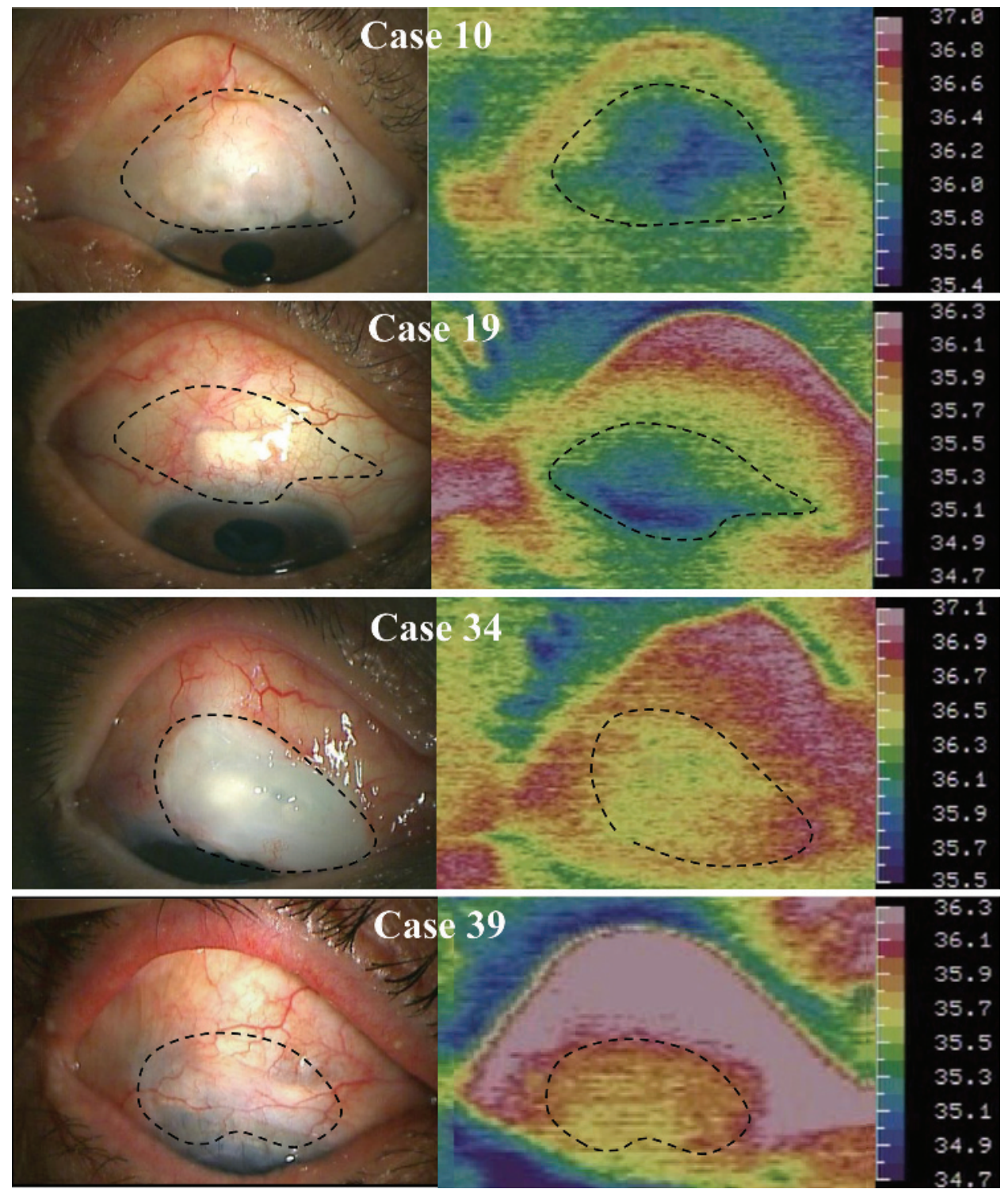

\section{Comparison of ocular surface temperature}

Ocular surface temperatures and statistical analyses of the good and poor IOP control groups are listed in table 2. There were significant differences between both the temperature of the filtering bleb and the TDB of the two groups. Figure 1 shows typical thermograms of the good (case 22 (fig 1A, B) and poor (case 35 (fig 1C, D)) IOP control groups. The temperature of the filtering bleb was clearly lower than that of the surrounding bulbar conjunctiva in the good IOP control group, while the temperature of the filtering bleb was almost the same as that of the surrounding bulbar conjunctiva in the poor control group.

\section{Effects of morphological appearance}

We examined the relationship of TDB to the morphological appearance of the filtering blebs classified based on the IBAGS ${ }^{3}$ (table 3). Statistical analyses showed no significant relationship between the TDB and any of the morphological factors. Figure 2 displays thermograms of cystic and flat blebs from each of the two groups. In the good IOP control group (cases 10 and 19), the temperature of the filtering bleb was lower than that of the surrounding bulbar conjunctiva, irrespective of its morphology. In the poor IOP control group (cases 39 and 34), the temperature of the filtering bleb was almost the same as that of the surrounding bulbar conjunctiva.

In addition, we examined the blebs to determine whether there were differences between the morphological appearances of the good and poor IOP control groups. Statistical analyses showed that the differences between the two groups were not significant (height $(\mathrm{H}) \mathrm{p}=0.1580$; extent (E) $\mathrm{p}=0.3872$; vasularity $(V) p=0.6043$; Seidel test $(S): p=0.7546$; MannWhitney $U$ test).

\section{DISCUSSION}

In the present study, we showed that the mean surface temperature of the filtering blebs in the good IOP control group was lower than that of the poor IOP control group. Furthermore, we introduced a thermographic index, TDB, to correct for body temperature, which varies among individuals. The TDB of the good IOP control group was significantly lower than that of the poor IOP control group. It could be argued that the morphological appearance, especially the vascularity, of the filtering bleb may affect its temperature. In this study, a thermographic measurement of each filtering bleb was made within $1 \mathrm{~s}$ after exposing the bleb, and as shown in table 3 , there 
Table 3 Relationship of TDB to the morphological appearance of the filtering blebs determined based on the IBAGS

\begin{tabular}{lrll}
\hline IBAGS grade & $\mathbf{n}$ & TDB $\left({ }^{\circ} \mathbf{C}\right)$ & p Value \\
\hline Height $(\mathrm{H})$ & & & \\
0 & 7 & $0.479(0.434)$ & $0.2231^{*}$ \\
1 & 13 & $0.285(0.198)$ & \\
2 & 12 & $0.454(0.199)$ & \\
3 & 7 & $0.471(0.152)$ & \\
Extent (E) & & & \\
0 & 8 & $0.438(0.418)$ & \\
1 & 9 & $0.344(0.228)$ & \\
2 & 15 & $0.433(0.214)$ & \\
3 & 7 & $0.386(0.146)$ & \\
Vascularity (V) & & & \\
0 & 8 & $0.462(0.173)$ & \\
1 & 13 & $0.469(0.196)$ & \\
2 & 8 & $0.269(0.300)$ & \\
3 & 10 & $0.385(0.321)$ & \\
Seidel test (S) & & & \\
0 & 33 & $0.411(0.263)$ & \\
1 & 6 & $0.375(0.219)$ & \\
\hline
\end{tabular}

IBAGS, Indiana Bleb Appearance Grading Scale; TDB, temperature decrease in the filtering bleb.

Values are mean (SD).

*ANOVA; †Mann-Whitney U test.

was no significant correlation between the TDB and the morphological appearance of the filtering blebs as determined by the IBAGS. ${ }^{3}$ Thus, the surface temperature of the filtering bleb was correlated with postoperative IOP control, regardless of whether the morphological appearance appeared flat or vascularised. These results imply that thermography may be useful to assess bleb function.

Some morphological approaches have been developed recently to assess bleb function. UBM has been widely used because it provides useful information through images of the scleral flap and its surrounding structure. However, assessments of bleb function using UBM are sometimes unreliable. ${ }^{10}$ In one study, ${ }^{7}$ the IOP was assessed as poor in six of 89 eyes with Type L blebs where the actual IOP was generally good. In another study, ${ }^{8}$ phacoemulsification significantly increased the IOP in eyes with filtering blebs, but the intrableb features did not change in the UBM image. Anterior segment OCT has been introduced to assess bleb function as a non-invasive examination. Generally, OCT shows that a functional bleb has a large hyporeflective space with a collection of fluid. Savini et al reported that hyporeflective fluid-filled spaces were not clearly visualised in two of 21 eyes with the good IOP control. ${ }^{9}$ Leung et al reported that subconjunctival fluid spaces could not be detected in two flattened blebs with good IOP control. ${ }^{10}$ Singh et al found that the majority of successful blebs demonstrated thickening of the bleb wall, but thickening was also found in six of 21 failed blebs. ${ }^{11}$ Thus, there are some limitations in the accuracy of these methods for assessing bleb function.

In a well functioning bleb, the aqueous humour flows continuously out of the scleral flap to perfuse the subconjunctival space around the filtering bleb. In a poorly functioning bleb, on the other hand, the amount of aqueous humour flowing into the filtering bleb is small, and therefore the aqueous humour turnover in the subconjunctival space is reduced. In a report by Heys et al, when the temperature of the ciliary body and the iris was specified to be $37.0^{\circ} \mathrm{C}$, the temperature of the aqueous humour near the cornea was calculated to be $34.4^{\circ} \mathrm{C} .^{24}$ Since aqueous humour cooler than the surrounding tissue is constantly flowing through a wellfunctioning bleb, this may cause the temperature of the cornea and filtering bleb to decrease to a level lower than that of the surrounding conjunctiva. On the other hand, a poorly functioning bleb is warmed by the eyelid and the conjunctiva, and therefore its temperature would be expected to remain the same as the surrounding tissue. Thermography may capture this phenomenon. Even if no cystic bleb is recognised in a slit-lamp examination, thermography can detect an area at the site of the scleral flap that is cooler than the surrounding bulbar conjunctiva, indicating that fresh aqueous humour may be flowing in the subconjunctival space around a filtering bleb (see case 19 in fig 2). Furthermore, even if a filtering bleb with a cystic appearance is present, thermography can show that the bleb is not functional; if an area cooler than the surrounding bulbar conjunctiva is not detected at the site of the scleral flap, this indicates that aqueous humour may not be flowing efficiently (see case 35 in fig 1 and case 39 in fig 2).

The TDB of each subject is shown in table 1. If the threshold level of the TDB for a well functioning bleb is defined to be $\geqslant 0.40^{\circ} \mathrm{C}$, there are 19 filtering blebs $(82.6 \%)$ in the good IOP control group and four filtering blebs $(25.0 \%)$ in the poor IOP control group. These results are significantly correlated with bleb function ( $p=0.0007$; Fisher' exact test). We examined the four exceptional filtering blebs in the poor IOP control group by UBM. The results of UBM suggested that two filtering blebs (case 26 and 39) were poorly functional (type E: encapsulated bleb); however the other filtering blebs (case 27 and 36) were fairly functional (type $\mathrm{H}$ : high-reflective bleb) according to Yamamoto's classification. ${ }^{7}$ Unfortunately, we are unable to explain why the IOP was elevated in the latter two cases, suggesting that similar to OCT and UBM, our thermographic method also has limitations. Thus, the efficiency of evaluating bleb function can be improved by using a combination of multiple examinations including OCT and UBM. In addition, improved software or more accurate instruments may be helpful to carry out more precise examinations. In this regard, we are developing an instrument that is specifically suitable for ocular surface thermography.

In conclusion, thermography sheds new insight on the evaluation of bleb function and may become another useful method for evaluating bleb function.

Competing interests: None declared.

Ethics approval: The procedures used in this study conformed to the guidelines adopted by the Declaration of Helsinki. The Institutional Review Board of the Ehime University Graduate School of Medicine and Minami Matsuyama Hospital approved the experimental protocol.

Patient consent: Obtained.

Provenance and peer review: Not commissioned; externally peer reviewed.

\section{REFERENCES}

1. Vesti E. Filtering blebs: follow up of trabeculectomy. Ophthalmic Surg 1993;24:24955.

2. Picht G, Grehn F. Classification of filtering blebs in trabeculectomy: biomicroscopy and functionality. Curr Opin Ophthalmol 1998;9:2-8

3. Cantor LB, Mantravadi A, WuDunn D, et al. Morphologic classification of filtering blebs after glaucoma filtration surgery: the Indiana Bleb Appearance Grading Scale. J Glaucoma 2003;12:266-71.

4. Hu CY, Matsuo H, Tomita G, et al. Clinical characteristics and leakage of functioning blebs after trabeculectomy with mitomycin-C in primary glaucoma patients. Ophthalmology 2003;110:345-52.

5. Migdal C, Hitchings R. The developing bleb: effect of topical antiprostaglandins on the outcome of glaucoma fistulising surgery. Br J Ophthalmol 1983;67:655-60.

6. Richter CU, Shingleton BJ, Bellows AR, et al. The development of encapsulated filtering blebs. Ophthalmology 1988;95:1163-8.

7. Yamamoto T, Sakuma T, Kitazawa Y. An ultrasound biomicroscopic study of filtering blebs after mitomycin C trabeculectomy. Ophthalmology 1995;102:1770-6 
8. Wang $\mathbf{X}$, Zhang $\mathrm{H}$, Li S, et al. The effects of phacoemulsification on intraocular pressure and ultrasound biomicroscopic image of filtering bleb in eyes with cataract and functioning filtering blebs. Eye 2009;23:112-16.

9. Savini G, Zanini M, Barboni P. Filtering blebs imaging by optical coherence tomography. Clin Experiment Ophthalmol 2005;33:483-9.

10. Leung CK, Yick DW, Kwong YY, et al. Analysis of bleb morphology after trabeculectomy with the Visante anterior segment optical coherence tomography. Br J Ophthalmol 2007:91:340-4.

11. Singh M, Chew PT, Friedman DS, et al. Imaging of trabeculectomy blebs using anterior segment optical coherence tomography. Ophthalmology 2007:114:47-53

12. Messmer EM, Zapp DM, Mackert MJ, et al. In vivo confocal microscopy of filtering blebs after trabeculectomy. Arch Ophthalmol 2006;124:1095-103.

13. Labbe A, Dupas B, Hamard P, et al. In vivo confocal microscopy study of blebs after filtering surgery. Ophthalmology 2005;112:1979-86.

14. Barnes RB. Thermography of the human body. Science 1963;140:870-7.

15. Mapstone R. Ocular thermography. Br J Ophthalmol 1970;54:751-4.
16. Kabayama T, Suzuki H, Horiuchi T, et al. Ganka Thermography No Kenkyu [Studies on thermography-its clinical application for ophthalmology]. Nippon Ganka Gakkai Zasshi 1979;83:326-35.

17. Efron N, Young G, Brennan NA. Ocular surface temperature. Curr Eye Res 1989:8:901-6.

18. Morgan PB, Soh MP, Efron N, et al. Potential applications of ocular thermography Optom Vis Sci 1993; 70:568-76.

19. Morgan PB, Tullo AB, Efron N. Infrared thermography of the tear film in dry eye. Eye 1995:9:615-8.

20. Mori A, Oguchi Y, Okusawa Y, et al. Use of high-speed, high-resolution thermography to evaluate the tear film layer. Am J Ophthalmol 1997;124:729-35.

21. Craig JP, Singh I, Tomlinson A, et al. The role of tear physiology in ocular surface temperature. Eye 2000;14:635-41.

22. Purslow C, Wolffsohn JS. Ocular surface temperature: a review. Eye Contact Lens 2005; 31:117-23.

23. Purslow $\mathbf{C}$, Wolffsohn J. The relation between physical properties of the anterior eye and ocular surface temperature. Optom Vis Sci 2007;84:197-201.

24. Heys JJ, Barocas VH. A boussinesq model of natural convection in the human eye and the formation of Krukenberg's spindle. Ann Biomed Eng 2002;30:392-401. 